\title{
No routine defibrillation threshold testing in all implantable cardioverter-defibrilator recipients - Still a question?
}

\author{
Dan Nicolae Tesloianu ${ }^{1}$, Andreea Maria Ursaru*,1, Radu Nicolae Ciudin ${ }^{2,3}$ \\ ${ }^{1}$ Interventional Laboratory, Cardiology Department, "Sf. Spiridon" University Emergency Hospital, lasi, \\ Romania, ${ }^{2} 4^{\text {th }}$ Clinic of Cardiology, "C.C. Iliescu" Institute of Cardiovascular Diseases, Bucharest, \\ Romania, "'C. Davila” University of Medicine and Pharmacy, Bucharest, Romania
}

\begin{abstract}
Nowadays the standard care for patients at high risk of sudden cardiac death (SCD) or life-threatening ventricular arrhythmias is the implantable cardioverter-defibrillator (ICD) and its variants (S-ICD, CRT-D). Although in the past ICD implantation was associated with routine defibrillation threshold (DFT) testing, recently more and more centers worldwide are abandoning DFT testing, considering the balance between the clinical benefit and increased procedural risks. In spite of this new approach, the usefulness/ suitability of DFT testing - the "to test or not to test" debate - still remains a matter of intense dispute among cardiologists. We present a brief history of ICDs and DFT testing along with the results of key/compelling studies on DFT testing and the management of patients with a high defibrillation threshold.
\end{abstract}

Keywords: defibrillation threshold testing, shock energy, arrhythmia cessation, implantable cardioverterdefibrillator

\section{Introduction}

In 1969 Michel Mirowski came to the Sinai Hospital in Baltimore to develop an automatic implantable defibrillator (AID), after his mentor and best friend, Prof. Dr. Harry Heller died unexpectedly, with a diagnosis of sudden cardiac death. 1970 marks the year in which Morton Mower and his collaborators published "Standby automatic defibrillator", which describes the key elements of such a device [1]. The first defibrillator was implanted in 1975 in a dog, and five years later in February 1980, the first AID was implanted in a 57-year-old woman with documented episodes of ventricular fibrillation unresponsive to therapy; post-implantation, recovered from a cardiac

Received: February 2017; Accepted after review: June 2017; Published: June 2017.

${ }^{*}$ Corresponding author: Andreea Maria Ursaru, MD, Cardiology Department, "Sf. Spiridon" University Emergency Hospital, 1, Independentei Blv, Iasi, Romania.

E-mail: andreea ursaru@yahoo.com arrest event following a myocardial infarction (Figure 1, left) [2].

The AIDs were relatively bulky (over 290 grams and a volume of over $150 \mathrm{ml}$ ), placed subcutaneously in the abdominal region and needed a median sternotomy/left lateral thoracotomy to fix the two patch electrodes on the epicardial surface; features such as pacing or other adjustable functions were unavailable at the time [3]. The second generation systems (AID-B) had bradycardia pacing and minimal programmability and the third generation systems, introduced in the early 1990s had antitachycardia pacing (ATP), low energy shocks for ventricular tachycardia (VT) treatment, extensive programmability and telemetry [4]. Since 1988 thoracotomy became unnecessary and the pacing lead system was placed transvenously [5], monophasic waves were no longer satisfactory for the transvenous approach, and which benefited the development of biphasic waveforms in 1991. The incorporation of an infraclavicular pulse generator, also known as a "hot can device" in 1993 together with biphasic shocks 
resulted in a considerable lowering of the DFT [6]. The evolution of the technical features of ICDs progressed steadily, the year 2004 marking the crucial/key moment when the Food and Drug Administration approved the

\section{$1^{\text {st }}$ device 1980}

$289 \mathrm{~g}, 150 \mathrm{cc}, 22 \mathrm{~mm}$

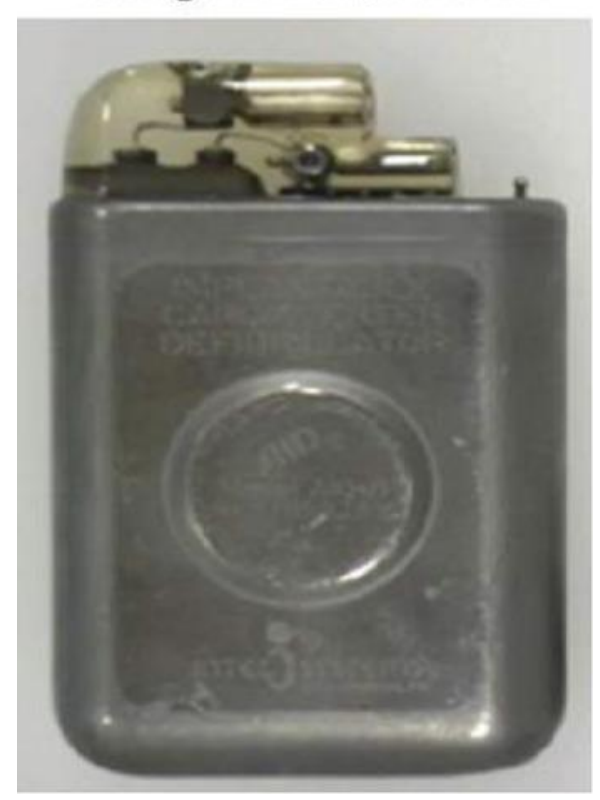

cardiac resynchronization therapy defibrillator (CRT-D) (Figure 1, right) as treatment for heart failure, followed by the validation of subcutaneous implantable cardioverter defibrillator (S-ICD) devices in 2012.

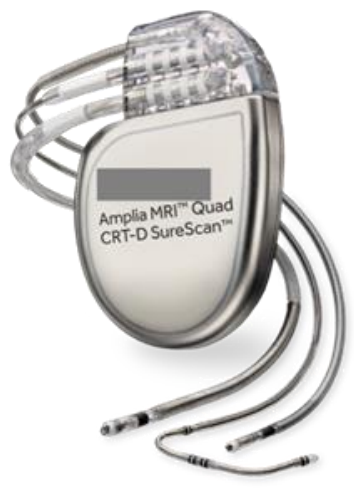

Fig. 1. Original implantable cardioverter defibrillator pulse generator, on the left, and a modern device on the right, CRT-D]

Since early experiences in ICD insertion led to sudden death due to ineffective defibrillation shock delivery, defibrillation threshold (DFT) testing became a standard of care for the elementary/primitive devices [7]. Contemporary systems are far less likely to fail defibrillation testing due to the new features: rapid charge times, high output devices routinely delivering $\geq 35$ Joules $(\mathrm{J}$ ), biphasic waveform shocks, active pectoral can as well as enhanced detection algorithms. Given these newly added features, there is increasing interest in minimizing the use of DFT testing at the time of implantation [8]. Still, there are specific clinical settings where defibrillation testing should be considered: concerns regarding the intraoperative integrity of the system, intrinsic sensed $R$ waves of $<5$ $\mathrm{mV}$, implantation in patients belonging to a group with higher incidence of elevated DFT (e.g. arrhythmogenic right ventricular dysplasia, hypertrophic cardiomyopathy
(HCM), left ventricular non-compaction, channelopathies - Brugada syndrome), rightsided or abdominal generator placement, implantation as a means of secondary prevention, S-ICD, in children and amiodarone treatment in the presence of an initially low defibrillation threshold margin on previous testing. On the other hand, typical contraindication for performing DFT are: artificial valve prosthesis or persistent atrial fibrillation without appropriate anticoagulation, left ventricular or left atrial/left atrial appendage thrombus, hemodynamic instability, significant aortic stenosis, severe chronic obstructive pulmonary disease, active coronary artery disease (CAD), percutaneous coronary intervention with stent placement or prior stroke in the past month, lack of critical care support or lack of informed consent [9, 10].

The risk of severe complications occurring in DFT testing appears to be rather small as 
seen in large studies of over 20.000 patients, but the possibility of underestimation of the actual rate is likely, due to: acute heart failure, acute respiratory failure, hypotension (due to anesthetics), acute myocardial dysfunction (especially in high energy testing), prolonged resuscitation, stroke or ischemic transitory attack, death, neurological post-resuscitation sequels, tissue necrosis with increased level of troponin post-testing [11-13].

It is important to differentiate between defibrillation testing and defibrillation margin testing. Intraprocedural defibrillation testing is a complex process that involves all the factors that participate in the detection and termination of a ventricular arrhythmia. These factors are: arrhythmia detection, appropriate sensing and adequate positioning of the shock vector so that the mass of myocardium is adequately encompassed the assessment of all parts of the ICD system, the ICD system viability defined by the capacity to terminate induced arrhythmia and the ICD integrity evaluated through high-voltage impedance. Defibrillation margin testing involves accurate detection of the safety margin, described as the adequate amount of delivered energy capable of terminating an induced arrhythmia, amount of energy that is below the maximal output energy of the device.

The term DFT designates the minimum to the minimum shock strength that is able to defibrillate. Historically, a threshold below a specific value has been used as an acceptable criteria for device implantation, and unlike the pacing threshold, the DFT is not an absolute value above which defibrillation will always be successful, but a value below which it will always fail. DFT is a term of probabilistic nature, because a successful shock of a given energy at the moment of testing does not guarantee the success of a shock of the same energy at any point in the future. However, even if predicting future success with complete accuracy is impossible, multiple successful shocks at a given energy do increase the likelihood of success [10]. A variety of methods have been used to determine DFT, methods that have evolved over time.

Defibrillation threshold testing implies repetitive ventricular fibrillation (VF) induction with decreased shock energy on each trial, until the shock energy is no longer able to terminate the arrhythmia - the "step-down protocol". In contrast, the "step-up protocol", translates as the gradual increase of shock energy until cessation of the arrhythmia; both protocols were used as pre-implantation testing methods in the early days of ICDs having the disadvantages being tedious and exposing patients to increased risks.

VF can be induced by three methods: shock discharge on the peak of the $T$ wave with a coupling interval of $200 \mathrm{~ms}$, after a pacing induced drive train of 5-8 beats of 400 ms cycle length; delivery of alternating current, several rates being available according to the manufacturers: $33 \mathrm{~Hz} / 30 \mathrm{~ms}$ (which means the $33 \mathrm{~Hz}$ burst induction delivers a rapid burst of VOO pacing pulses to the ventricle and the pacing interval is fixed at $30 \mathrm{~ms}$ ), $50 \mathrm{~Hz} / 20 \mathrm{~ms}$ or $20 \mathrm{~Hz} / 50 \mathrm{~ms}$; delivery of high rate, high output pulses through the shocking electrode with 2 different cycle lengths.

Defibrillation margin testing involves successful defibrillation at a specific energy level shock, below the maximum limit for the device; if the first shock is successful, then this energy level can be repeated once or twice more (single energy success method). This second technique has the advantage of a minimal testing to establish adequate acute defibrillation efficacy. For this method, as well as in the case of the "step-down" protocol, the first shock is typically set at a value of at least $10 \mathrm{~J}$ less than the maximal output of the device $[9,14]$.

Upper limit of vulnerability (ULV) is the third method of defibrillation testing that reduces or even eliminates the need of VF induction. Multiple $\mathrm{T}$ wave shocks are delivered and the weakest shock strength at which VF is no longer induced is measured; this energy has been shown to correlate closely with the defibrillation threshold; usually $U L V \leq 15 \mathrm{~J}$ strongly correlates with a DFT $\leq 20$ $\mathrm{J}$ (thus VF is induced in only $20-40 \%$ of the tested patients (reducing the patient safety risks); a big disadvantage is that this method is not involving the assessment of sensing [15].

The DFT testing procedure brings maximum benefits for patients belonging to a high DTF expectancy category to the categories with high DFT expectancy at 
testing. High DFT is defined as an absolute value of shock energy $>25 \mathrm{~J}$ (device with $35 \mathrm{~J}$ maxim output) or a safety margin of $<10 \mathrm{~J}$ below the maximum output of the device.

Non-invasive strategies in management of high DFT includes: measuring the impedance and confirming all the connections; changing of polarity - changing the RV coil from anode (as usual) to a cathodal RV coil with a possible reduction of $10-15 \%$ in DFT; biphasic waveform tilt optimization, where manufacturers allow this option [16]; medication, Sotalol and Dofetilide, are reported to reduce DFT $[17,18]$. A notable mention are the preventable causes of high DFT: medication (lidocaine, mexiletine, moricizine, verapamil, venlafaxine, anesthetic agents - including propofol necessary for DFT testing, cocaine, sildenafil and amiodarone in chronic administration -acute i.v. can reduce DFT), acidosis, hypoxia, anemia, electrolyte abnormalities (hypomagnesemia, hypocalcemia, hyperkalemia), post-procedure pneumothorax or large pleural effusions, prolonged anesthesia or time of testing (myocardial stunning, polarization modification produced through repetitive shocks) [18].

Invasive strategies in management of high DFT identify: use of high output ICD device, altering the shock vector through RV lead repositioning, manipulation of the superior vena cava coil, additional coil implantation in the azygous vein, coronary sinus and epicardial space or addition of subcutaneous arrays, the latter rarely used nowadays [18, 19].

Beginning with the early 2000s', electrophysiologists started questioning the benefits of defibrillation threshold testing, and over time, the "not to test" arguments became dominant, recently supported by data from clinical trials with a large number of patients. [11, 20-25].

The most common reasons for avoiding DFT testing nowadays are: Nowadays, reasons for abandoning the DFT testing are: increased incidence of VT over VF, an arrhythmia that needs significantly lower energy for cardioversion and can sometimes responds to ATP or even terminate spontaneously. Even in the much less likely event of VF, the probabilistic nature of DFT suggests that if repeated several times, shocks inferior to the determined threshold have the possibility of terminating the arrhythmia. So, in a patient with high DFT who does receive an ICD, the failure to terminate VF at the time of testing does not mean that the patient will succumb to an episode of spontaneous VF [20]. Moreover $40 \%$ of ICDs implanted for primary prevention will not encounter sustained ventricular arrhythmias until the end-of-battery-life; VFs are not all the same. While induced VF model ( $T$-wave shock or with a short burst of $50-\mathrm{Hz}$ AC current) can be replicated through electrocution or commotio-cordis, spontaneous VF usually occurs due to myocardial ischemia [26]. The manner in which occurs is important due to the fact that induced VF has lower rates and a higher degree of regularity, which correlates with a higher probability of effective defibrillation. Different methods of inducing VF are correlated with different DFTs. Low DFTs during ICD implantation do not guarantee the termination of spontaneous VF. Failure to terminate an induced VF during testing does not necessarily predict failure to terminate a spontaneous sustained ventricular arrhythmia. The very low sudden-death rate in patients with implanted ICD is not necessarily attributable to DFT testing and performing the test adds little information, as the patient's probability to have a successful DFT is very high (in more than 800 consecutive patients in SCD-HeFT study, the rate of acceptable DFT values was of $84 \%)[23,27]$. Another factor that must be considered in recommendations regarding DFT testing is the risk associated with this practice [11]. Using high energy shocks has the advantage of the increased likelihood of successful terminating VT/VF and SVT with no additional discomfort (the pain/discomfort felt by the patient is almost identical for a shock energy value of $5 \mathrm{~J}$ as for one of $40 \mathrm{~J}$ ). This advantage, along with the risk of transforming VT into VF, present at low energy shocks, point out that a high energy shock is more useful, and in this case, that DTF testing becomes unnecessary [28].

The first large trial which clearly stated against DFT testing in ICD recipients was SCD-HeFT (Sudden Cardiac Death in Heart Failure Trial): a primary prevention ICD trial 
that compared single-chamber ICDs with amiodarone or placebo in improving survival in patients with symptomatic heart failure and left ventricular ejection fractions $\leq 35 \%$ [29]. The sub-study by Blatt et al. included 811 ICD implanted patients, (baseline DFT data being available for 717 patients $(88.4 \%)$ ), the conclusions being that there was no difference in survival between patients who had a low DFT (DFT $\leq 10 \mathrm{~J}$ ) and those who had a high DFT (DFT > 10J); $83 \%$ of the spontaneous events benefited of first-shock efficacy with no significant difference according to baseline DFT. Thus, DFT testing was shown to bring no additional benefits.

The results of this early trial were clearly reinforced by the more recent two ones dedicated to prospective assessment of DFT testing utility: SIMPLE and NORDIC ICD. SAFE-ICD trial, in 2012, already "indicated a limited clinical relevance for DFT testing, thus supporting a strategy of omitting DFT during an ICD implant" in a prospective observational study designed to evaluate the outcome of 2 strategies: performing defibrillation testing $(D T+)$ versus not performing defibrillation testing (DT-) during de novo ICD implants in 2120 consecutive patients (836 DT+ and 1,284 DT-) [30, 31].

SIMPLE (Shockless IMPLant Evaluation Trial) was a randomized comparison 1:1 between defibrillation margin testing (test all) and no defibrillation margin testing (test none) that included 2500 patients with initial transvenous ICD implantation. The risk of arrhythmic death or ineffective ICD shock was similar in the test all and test none groups and the risk of procedure related adverse events was also similar in both groups; the study has included a reduced number of patients with high DFT probability: $4 \%$ had hypertrophic cardiomyopathy and 1\% non-ipsilateral generator placement; patients with channelopathies, sarcoidosis and congenital heart disease have been excluded from the study group. Patients with subcutaneous ICDs were also excluded and upper limit of vulnerability, a potentially safer method of testing was not used. The SIMPLE authors asserted that ICD implantation without defibrillation testing is non-inferior to implantation with testing and that for most patients receiving their first ICD, a strategy of implantation without defibrillation testing should be preferred [32].

NORDIC ICD (The NO Regular Defibrillation testing In Cardioverter Defibrillator Implantation trial) was a 1:1 randomized trial to first time ICD implantation with or without DFT testing that included 1077 patients. The intra-operative DF testing was standardized across all participants: if the initial shock of $15 \mathrm{~J}$ was successful, DFT testing was terminated; if unsuccessful, a second shock $24 \mathrm{~J}$ was delivered, and had to be confirmed; if the shocks were successful twice, DFT testing was terminated; if unsuccessful, a revision of the system was recommended, and the DFT testing was repeated. All ICD shocks were programmed to $40 \mathrm{~J}$ irrespective of DFT test. Two hundred one serious adverse events in 168 patients of which 89 events occurred in 74 patients without DF testing and one hundred twelve events occurred in 94 patients with DFT testing within 30 days of follow-up. There was only one significant difference between the two groups (DFT-testing and no-DFT testing) regarding the serious adverse events: intraoperative hypotension, which did not occur in those without DFT testing. This study concluded that: "Defibrillation efficacy during follow-up is not inferior in patients with a $40 \mathrm{~J}$ ICD implanted without DF testing. Defibrillation testing during first time ICD implantation should no longer be recommended for routine left-sided ICD implantation" [33].

The recommendations of 2015 Consensus Statement on Optimal ICD Programming and Testing [34] concerning the intraprocedural testing of defibrillation efficacy are exposed in Table 1. 
Table 1. Intraprocedural testing of defibrillation efficacy recommendations states

\begin{tabular}{|c|c|c|}
\hline $\begin{array}{l}\text { Intraprocedural Testing of Defibrillation Efficacy } \\
\text { Recommendations }\end{array}$ & $\begin{array}{l}\text { Class of } \\
\text { recommendation }\end{array}$ & $\begin{array}{l}\text { Level of } \\
\text { Evidence }\end{array}$ \\
\hline $\begin{array}{l}\text { Defibrillation efficacy testing is recommended in patients } \\
\text { undergoing a subcutaneous ICD implantation. }\end{array}$ & 1 & C-LD \\
\hline $\begin{array}{l}\text { It is reasonable to omit defibrillation efficacy testing in patients } \\
\text { undergoing initial left pectoral transvenous ICD implantation } \\
\text { procedures where appropriate sensing, pacing, and impedance } \\
\text { values are obtained with fluoroscopically well-positioned RV leads. }\end{array}$ & IIA & $B-R$ \\
\hline $\begin{array}{l}\text { Defibrillation efficacy testing is reasonable in patients undergoing a } \\
\text { right pectoral transvenous ICD implantation or ICD pulse generator } \\
\text { changes. }\end{array}$ & IIA & B-NR \\
\hline $\begin{array}{l}\text { Defibrillation efficacy testing at the time of implantation of a } \\
\text { transvenous ICD should not be performed on patients with a } \\
\text { documented non chronic cardiac thrombus, atrial fibrillation or } \\
\text { atrial flutter without adequate systemic anticoagulation, critical } \\
\text { aortic stenosis, unstable CAD, recent stroke or TIA, hemodynamic } \\
\text { instability, or other known morbidities associated with poor } \\
\text { outcomes }\end{array}$ & III & C-LD \\
\hline
\end{tabular}

Finally, a meta-analysis was performed using 6 databases, including 13 studies and a total of 9740 patients. No significant differences between DFT versus no-DFT cohorts were found concerning all-cause mortality, composite end point of implantable cardioverter-defibrillator efficacy (arrhythmic deaths and ineffective shocks), and composite safety end point (the sum of complications recorded at 30 days). It is important to note that certain patient populations were underrepresented in the meta-analysis. These include HCM, channelopathies - especially Brugada syndrome, congenital heart disease, right-sided device implants, ICD implant for secondary prevention and ICD generator replacement. In conclusion, "systematic review of contemporary data suggests a modest average effect of DFT, if any, in terms of mortality, shock efficacy, or safety. Therefore, DFT testing seems to no longer be compulsory during de novo implantation. However, DFT testing may still be clinically relevant in specific patient populations." [35].

The benefits of DFT testing can be summarized for a small and specific patient category, as noted above: patients undergoing a subcutaneous ICD implantation, patients undergoing a right pectoral transvenous ICD implantation, ICD pulse generator changes or ICD implant for secondary prevention, ICD implantation in HCM, channelopathies especially Brugada syndrome, congenital heart disease. In 2017, Francia et al have retrospectively analyzed a cohort of $66 \mathrm{HCM}$ patients implanted with an ICD and DFT was determined in $25(38 \%)$ of these patients. Despite the fact that young age and massive hypertrophy affected the decision of DFT testing, the authors concluded that "contemporary ICDs are safe and effective in HCM patients independently from the magnitude of LVH. DFT testing does not predict shock efficacy for spontaneous VT/VF." [36]. Previously, in 2015, a similar conclusion was stated by Ashino et al. that included a number of only $11 \mathrm{HCM}$ patients with ICD implantation. Additionally this study proved no significant difference between DFT and nonDFT testing in ICD recipients, taking into consideration the underlying cardiac conditions of the patients: coronary artery disease, idiopathic ventricular arrhythmia, dilated cardiomyopathy, valvular disease, Brugada syndrome (from a total number of 18 patients, 16 underwent a DFT testing, DFT testing being commonly performed in cases of 
Brugada syndrome) and in secondary prevention [37].

Data from the Israeli ICD Registry, probably the biggest one dedicated to DFT testing, provided similar conclusions regarding the ICD recipients with idiopathic ventricular arrhythmia, HCM, in secondary prevention and in patients using antiarrhythmic medication. Also, from a total number of 27 Brugada syndrome patients, more than a half had no DFT testing and no significant differences in the incidences of mortality, malignant ventricular arrhythmias, and inappropriate ICD discharges between patients who underwent DFT testing and those who did not were observed [38].

In his article based on a close analysis of SIMPLE study James Gamble supports avoiding DFTs both in primary as well as in secondary prevention patients [39].

Modi et al. presented at the 15th World Congress in Heart Disease, Annual Scientific Session 2010 - Canada, a retrospective study reviewing 144 patients with ICD generator replacement concluding that DFTs tend to remain stable in time, no intervention being needed after DFT testing. Therefore routine DFT testing during ICD generator replacement

\section{References}

1. Mirowski M, Mower MM, Staewen WS, Tabatznik B, Mendeloff Al. Standby automatic defibrillator. An approach to prevention of sudden coronary death. Arch Intern Med 1970; 126:158-161.

2. Mirowski M, Reid PR, Mower MM, et al. Termination of malignant ventricular arrhythmias with an implanted automatic defibrillator in human beings. $N$ Engl $J$ Med 1980; 303:322-324.

3. Watkins $L$ Jr, Mirowski M, Mower MM, et al. Automatic defibrillation in man. The initial surgical experience. J Thorac Cardiovasc Surg 1981; 82:492-500.

4. Furman S, Kim S. The present status of implantable cardioverter defibrillator therapy. $J$ Cardiovas Electrophysiol 1993; 3:602-625.

5. Bardy GH, Johnson G, Poole JE, et al. A simplified, single-lead unipolar transvenous cardioversion-defibrillation system. Circulation 1993; 88:543-547. may not be necessary and thus the perioperative risk can be avoided [40]. Earlier, in 2008, similar conclusion resulted from a study of 209 retrospectively reviewed patients with ICD generator replacement. Only three of these patients had high DFT, in all cases the factors suggesting the need for DFT testing being identified prior to testing [41].

\section{Conclusions}

Although in the past ICD implantation was associated with routine DFT, recently more and more centers worldwide are abandoning DFT testing, considering the balance between the clinical benefit and increased procedural risks. In spite of this new approach, the usefulness/ suitability of DFT testing - the "to test or not to test" debate - still remains a matter of intense dispute among cardiologists.

\section{Competing interests}

The authors declare that they have no competing interests.
6. Kavanagh K, Tang A, Rollins D, Smith W, Ideker R. Comparison of the internal defibrillation thresholds for monophasic and double and single capacitor biphasic waveforms. J Am Coll Cardiol 1989, 14:13431349.

7. Echt DS, et al. Clinical experience, complications, and survival in 70 patients with the automatic implantable cardioverter/defibrillator. Circulation 1985; 71:289.

8. Russo AM, Chung MK. Is defibrillation testing necessary for implantable transvenous defibrillators? defibrillation testing is necessary at the time of implantable cardioverter defibrillator implantation. Circ Arrhythm Electrophysiol 2014; 7:337-346.

9. Rizkallah J, Exner DV. Clinical cardiac pacing, defibrillation and resynchronization therapy, $5^{\text {th }}$ Ed. Elsevier 2017; 718-739.

10. Hayes D, Asirvatham $S$, Friedman $P$, et al. Cardiac pacing defibrillation and 
resynchronization. A clinical approach. Third Edition, 2013.

11. Birnie D, Tung S, Simpson $C$ et al. Complications associated with defibrillation threshold testing: The Canadian experience. Heart Rhythm 2008, 5:387-390.

12. Bänsch D. Defibrillation testing during defibrillator implantation. Arrhythmia \& Electrophysiology Review 2012; 1:51-53.

13. Vamos M, Healey JS, Wang J. Troponin levels after ICD implantation with and without defibrillation testing and their predictive value for outcomes: Insights from the SIMPLE trial. Heart Rhythm 2016; 13:504-510.

14. Kempa M, Królak $T$, Drelich $L$, et al. Predischarge defibrillation testing: Is it still justified? Cardiology Journal 2016, 23:532538.

15. Birgersdotter-Green U, Undesser K, Fujimura $\mathrm{O}$, et al. Correlation of acute and chronic defibrillation threshold with upper limit of vulnerability determined in normal sinus rhythm. J Interv Card Electrophysiol 1999; 3:155-161.

16. Zienciuk A, Lubiński A, Królak T et al. Effects of shock polarity reversal on defibrillation threshold in an implantable cardioverterdefibrillator. Kardiol Pol 2007; 65:495-500.

17. Simon RD, Sturdivant JL, Leman RB, Wharton JM, Gold MR. The effect of dofetilide on ventricular defibrillation thresholds. Pacing Clin Electrophysiol 2009; 32:24-28.

18. Kanjwal K, Mainigi S. Defibrillation Threshold Testing: A primer. J Innov Card Rhythm Manag 2012; 3:5-15

19. Kommuri N, Kollepara SL, Saulitis E, Siddiqui MA. Azygos vein lead implantation for high defibrillation thresholds in implantable cardioverter defibrillator placement. Indian Pacing Electrophysiol J 2010; 10:49-54.

20. Strickberger $S A$, Klein $\mathrm{G}$. Is defibrillation testing required for defibrillator implantation? J Am Coll Cardiol 2004; 44:88-91.

21. Russo AM, Sauer W, Gerstenfeld EP et al. Defibrillation threshold testing: is it really necessary at the time of implantable cardioverter-defibrillator insertion? Heart Rhythm 2005; 2:456-461.

22. Swerdlow CD, Russo AM, Degroot PJ. The dilemma of ICD implants testing. Pacing Clin Electrophysiol 2007; 30:675-700.

23. Viskin S, Rosso R. The top 10 reasons to avoid defibrillation threshold testing during ICD implantation. Heart Rhythm 2008; 5:391-393

24. Markowitz SM. To test or not to test during defibrillator implantation? A reassessment of the conventional wisdom. J Cardiovasc Electrophysiol 2008; 19:406-408.
25. Gula LJ, Massel D, Krahn AD et al. Is defibrillation testing still necessary? A decision analysis and Markov model. J Cardiovasc Electrophysiol 2008; 19:400-405.

26. Taneja T, Goldberger J, Johnson D, et al. Is all ventricular fibrillation the same? (Influence of mode of induction on characteristics of ventricular fibrillation). $J$ Cardiovasc Electrophysiol 2000; 11:1355-1363.

27. Bardy G, Lee K, Mark D. Amiodarone or an implantable cardioverter-defibrillator for congestive heart failure. N Engl J Med 2005; 352:225-237.

28. Madhavan M, Friedman P. Optimal programming of implantable cardiacdefibrillators. Circulation 2013; 128:659-672

29. Bardy GH, Lee K., Mark DB, et al. Amiodarone or an implantable cardioverter-defibrillator for congestive heart failure. N Engl J Med 2005; 352:225-237

30. Blatt J, Poole J, Johnson Get al. No benefit from defibrillation threshold testing in the SCDHeFT (Sudden Cardiac Death in Heart Failure Trial). J Am Coll Cardiol 2008; 52: 551-556;

31. Brignole $M$, Occhetta $E$, Bongiorni $M G$ et al. Clinical evaluation of defibrillation testing in an unselected population of 2,120 consecutive patients undergoing first implantable cardioverter-defibrillator implant. J Am Coll Cardiol 2012; 11:981-987.

32. Healey J, Hohnloser S, Glikson $M$ et al. Cardioverter defibrillator implantation without induction of ventricular fibrillation: a singleblind, non-inferiority, randomised controld trial (SIMPLE) Lancet 2015; 385:785-791.

33. Bänsch D, Bonnemeier $\mathrm{H}$, Brandt $\mathrm{J}$ et al. Intraoperative defibrillation testing and clinical shock efficacy in patients with implantable cardioverter-defibrillators: the NORDIC ICD randomized clinical trial. Eur Heart $J$ 2015; 36:2500-2507.

34. Wilkoff B, Fauchier L, Stiles M, et al. 2015 HRS/EHRA/APHRS/SOLAECE expert consensus statement on optimal implantable cardioverter-defibrillator programming and testing. Heart Rhythm 2016; 13(2):e50-86.

35. Phan $\mathrm{K}, \mathrm{Ha} \mathrm{H}$, Kabunga $P$, et al. Systematic review of defibrillation threshold testing at de novo implantation. Circ Arrhythm Electrophysiol 2016; 9:e003357.

36. Francia P, Adduci C, Semprini L. Prognostic implications of defibrillation threshold testing in patients with hypertrophic cardiomyopathy. $J$ Cardiovasc Electrophysiol 2017; 28:103-108.

37. Ashino S, Nakai $T$, Sonoda $K$, et al. Assessment of efficacy and necessity of routine defibrillation threshold testing in patients undergoing Implantable Cardioverter- 
Defibrillator (ICD) implantation. Int Heart $J$ 2015; 56:618-621.

38. Arnson $Y$, Suleiman $M$, Glikson $M$ et al. Role of defibrillation threshold testing during implantable cardioverter-defibrillator placement: data from the Israeli ICD Registry. Heart Rhythm 2014; 11:814-821.

39. Gamble J. Shall we do a DFT? A clinical review of defibrillation threshold testing in 2014. British Heart Rhythm Society Editorial, 2014.
40. Modi $\mathrm{HH}$, Aeriachaipanich A, Bhan A, Duggal $M$. Routine DFT testing: is it necessary during ICD generator replacement? In Proceedings of 15th World Congress in Heart Disease, Annual Scientific Session 2010, Canada.

41. Singh M, Akar J, Cytron J. Value of routine DFT testing at time of ICD pulse generator replacement. In Proceedings of 14th World Congress in Heart Disease, Annual Scientific Session 2008, Canada. 